\title{
On the Chacteristic Numbers of Voting Games
}

\author{
MATHIEU MARTIN \\ THEMA, Departments of Economics \\ Université de Cergy Pontoise, 33 Boulevard du Port, \\ 95011 Cergy Pontoise cedex, France. \\ e-mail: mathieu.martin@eco.u-cergy.fr \\ VINCENT MERLIN* \\ CNRS and CREM, University of Caen \\ MRSH 230, Esplanade de la Paix \\ 14032 Caen cedex, France. \\ e-mail:merlin@econ.unicaen.fr
}

\begin{abstract}
This paper deals with the non-emptiness of the stability set for any proper voting game. We present an upper bound on the number of alternatives which guarantees the non emptiness of this solution concept. We show that this bound is greater than or equal to the one given by Le Breton and Salles [6] for quota games.
\end{abstract}

Keywords: voting game, core, stability set JEL classification: C7,D7.

\section{Introduction}

The concept of voting games is of crucial importance for social choice theory as well as game theory. A voting game is characterized by a set of individuals and by the set of all winning coalitions, that is, groups of individuals which can enforce a decision. Then, knowing the preferences of the voters, an alternative belongs to the core of a voting game if the members of any winning coalition never prefer unanimously another proposition.

\footnotetext{
* Corresponding author. MRSH, bureau 230, Université de Caen, Esplanade de la Paix, 14032 Caen cedex, France. e-mail: merlin@econ.unicaen.fr. Tel: +33 (0) 231 5662 49. Fax: +33 (0) 231565513
} 
Unfortunately, it is easy to prove that the core of a voting game may be empty, just by taking this simple example due to Condorcet [1].

Example 1. Consider three voters, 1, 2, and 3 who have to choose among three alternatives, $x, y$ and $z$. A coalition is winning if it contains at least two voters. The notation $x \succ_{i} y \succ_{i} z$ will mean that voter $i$ prefers $x$ to $y$, and $y$ to $z$; The fact that two alternatives $x$ and $y$ are considered as equivalent by voter $i$ will be denoted by $x \sim_{i} y$. Let assume that the preferences of the voters are the following ones:

$$
x \succ_{1} y \succ_{1} z, z \succ_{2} x \succ_{2} y, y \succ_{3} z \succ_{3} x
$$

In this case, voters 1 and 2 prefer $x$ to $y$, voters 2 and 3 prefer $z$ to $x$, and voters 1 and $3, y$ to $z$ : The core of this game is empty.

A way out of this "paradox of voting" has been suggested by Rubinstein [13]. His objective is to circumvent the possible cyclicity of the dominance relation obtained from head to head comparisons by assuming that voters may be, in some sense, prudent. The behavior he intends to model is the following one:

"True, I prefer $b$ to $a$, but if $b$ is adopted, then a situation arises where the majority prefers $c$. Since $c$ is worse than $a$ from my point of view, I will not take any chances and will not vote for $b$ in place of $a . "$

Assuming this prudent behavior in the Condorcet example leads to the conclusion that no winning coalition would form, and any alternative is a stable status quo. Rubinstein [13] shows that this is a generic result. As long as voters preferences are strict (without indifference), there always exists a non-empty subset of stable alternatives under this behavior: It is called the stability set.

Unfortunately, as soon as indifference among candidates is allowed in the individual preferences, Le Breton and Salles have shown that this possibility result collapses [6]. 
Example 2. Consider 5 voters, with the following preferences over 5 alternatives:

$$
\begin{aligned}
& a \succ_{1} b \sim_{1} e \succ_{1} c \succ_{1} d \\
& e \succ_{2} a \sim_{2} d \succ_{2} b \succ_{2} c \\
& d \succ_{3} e \sim_{3} c \succ_{3} a \succ_{3} b \\
& c \succ_{4} d \sim_{4} b \succ_{4} e \succ_{4} a \\
& b \succ_{5} c \sim_{5} a \succ_{5} d \succ_{5} e
\end{aligned}
$$

Considering the majority game, $a$ beats $b$ via the coalition $C_{1}=$ $\{1,2,3\}$, and $e$ beats $a$ via $C_{2}=\{2,3,4\}$. The alternative $b$ will belong to the stability set if one voter in $C_{1}$ prefers $b$ to $e$. As players 2 and 3 prefer $e$ to $b$ and player 1 is indifferent between $b$ and $e$, there is no cost for forming coalition $C_{1}$. Thus $b$ is dominated, and a similar reasoning holds for any other alternative; The stability set is empty.

Le Breton [5], Le Breton and Salles [6], Li [7] and Martin [8] have tried to find a necessary and sufficient condition that guarantees the non emptiness of the stability set. Their investigations seek a result similar to Nakamura's theorem [11] which states that the core is non empty for any preference profile if and only if the number of alternatives is strictly lower than the Nakamura number, $\nu$, that can be computed for any voting game. Le Breton and Salles and Li already obtained preliminary results for the stability set by using bounds related to the Nakamura number. We prove here that if a "Nakamura like theorem" exists for the stability set, it should depend on a new number, $\theta$. Our main result shows that $\theta$ is an upper bound on the number of alternatives which guarantees the non emptiness of this solution concept. Moreover, this bound is greater than or equal to the one given by Le Breton and Salles [6] for any voting games.

The paper is organized as follows. Section 2 is devoted to the notation and definitions. In Section 3, we review the main results on the existence of the core and stability set. The new number is defined in Section 4. We show with examples that it is different from the one proposed by Le Breton and Salles, and prove some new results. Section 5 is devoted to concluding comments and raises some open issues. 


\section{Basic notation and definitions}

Let $N=\{1,2, \ldots, n\}$ be a finite set of $n$ individuals and $X=$ $\left\{x_{1}, x_{2}, \ldots, x_{k}\right\}$ be a finite set of $k$ alternatives. For each $i \in N$, the preference of person $i$ is a binary relation $\succsim_{i}$ on $X$ which is reflexive, connected and transitive. $\succ_{i}\left(\right.$ resp. $\left.\sim_{i}\right)$ represents the asymmetric (resp. symmetric) component of $\succsim_{i}$. Such a binary relation is called a complete preorder.

$\mathcal{R}^{n}$ is the $\mathrm{n}$-fold Cartesian product of the set of individual preferences. An element of $\mathcal{R}^{n},\left(\succsim_{)}=\left(\succsim_{1}, \succsim_{2}, \ldots, \succsim_{n}\right)\right.$ is called a profile. Given a set $Y,|Y|$ is the number of elements in $Y$.

This paper deals with voting games, that is some pairs $G=$ $(N, W)$ where $W$ is the set of winning coalitions of $G$ (non empty subsets of $N$ ) satisfying a monotonicity condition: $S \in W$ and $S \subset T$ implies $T \in W$. A winning coalition $S$ is called a minimal winning coalition if and only if, $S-\{i\} \notin W, \forall i \in S$. In this paper, we only deal with proper voting games, that is games such that there do not exist two disjoint winning coalitions. In the literature, a class of voting games is particularly studied, the quota games. A quota game, denoted by $G(n, q)$ ( $q$ for quota), is a voting game such that $C \in W$ if and only if $|C| \geq q$. A weighted game $G\left(w_{1}, \ldots, w_{n} ; q\right)$ assigns $w_{i}$ votes to player $i$; A coalition is winning if and only if its members gather more than $q$ votes.

The following definition, due to Nakamura, is of crucial importance in the study of voting games.

Definition 1. The Nakamura number of a voting game $G=(N, W)$ is the integer $\nu(G)$ defined as follows:

(i) If $\bigcap_{C \in W} C \neq \emptyset, \nu(G)=\infty$,

(ii) If $\bigcap_{C \in W} C=\emptyset, \nu(G)=\min \left\{|\sigma|: \sigma \subseteq W\right.$ et $\left.\bigcap_{C \in \sigma} C=\emptyset\right\}$

In words, the Nakamura number is the minimal number of winning coalitions such that their intersection is empty. In the case where an individual belongs to all these coalitions (this individual is called a vetoer), we assume that the Nakamura number tends to infinity.

To complete the description of the social decision problem, we can define two collective dominance relations, $\succ$ and $\succ \succ$, and two associated solution concepts, the core and the stability set. 
Definition 2. The alternative $y \in X$ dominates the alternative $x \in$ $X$ via the coalition $C$ given $(\succsim) \in \mathcal{R}^{n}$, if $C \in W$ and $y \succ_{i} x \forall i \in C$. This will be denoted $y \succ_{C} x . y$ dominates $x$ if there exists $C \in W$ such that $y \succ_{C} x$; this will be denoted by $y \succ x$.

Definition 3. The core of a voting game $G(N, W)$, given the profile $(\succsim) \in \mathcal{R}^{n}$, is the set of alternatives which are not dominated by another alternative,i.e.,

$$
\operatorname{Cor}(G,(\succsim))=\{x \in X: \nexists y \in X \text { such that } y \succ x\}
$$

The other dominance relation and its associated concept of solution are due to Rubinstein [13]. This relation is close to the covering relations introduced by Miller [9] or Gillies [3].

Definition 4. The alternative $y \in X$ dominates alternative $x \in X$ in order one, via the coalition $C$, denoted by $y \succ_{C} x$, if

(i) $C \in W$

(ii) $y \succ_{C} x$

(iii) $z \succsim_{i} x$ for all $i \in C$ and all $z \in X$ for which $z \succ y$.

$y$ dominates $x$ in order one, denoted by $y \succ \succ x$, if there is a coalition $C \in W$ for which $y \succ_{C} x$.

Definition 5. The stability set of the game $G(N, W)$ for the profile $(\succsim) \in R^{n}$ is the set of alternatives which are not dominated in order 1 by another alternative, i.e,

$$
S(G,(\succsim))=\{x \in X: \nexists y \in X \text { such that } y \succ \succ x\}
$$

Clearly, if an alternative belongs to the core, then it belongs to the stability set (obviously, the converse is not true). Therefore, for a given profile, the core is a subset of the stability set.

Remark 1. If a binary relation over a finite set is acyclic, then the set of maximal elements for this relation is non-empty. Conversely, if the set of maximal elements is empty, then there is a cycle.

\section{Preliminary results}

The main result on the existence of the core is the following one: 
Theorem 1 (Nakamura, [11]). Let $G(N, W)$ be a voting game.

$$
\operatorname{Cor}(G,(\succsim)) \neq \emptyset \quad \forall(\succsim) \in \mathcal{R}^{n} \Longleftrightarrow k<\nu(G)
$$

The Nakamura number is the upper bound on the number of alternatives which guarantees the non emptiness of the core. If the number of alternatives is greater than the Nakamura number, then there exists at least one profile such that the core is empty. In the particular case of quota games, this bound is equal to $\left\lceil\frac{n}{n-q}\right\rceil^{1}$ (this result is due to Ferejohn and Grether [2], Peleg [12] and Greenberg [4]). A simple proof of the equivalence between the number $\left\lceil\frac{n}{n-q}\right\rceil$ and the Nakamura number for quota games is given by Moulin [10].

The second result is due to Rubinstein [13] and, in contrast to the Nakamura's result, is very optimistic.

Theorem 2 (Rubinstein, [13]). The stability set is always nonempty when the individual preferences are linear orders, that is, when indifference is not allowed.

This result solves the well-known paradox of voting by introducing a notion of farsightedness (see the introduction for Rubinstein's motivations) in the individual preferences. Unfortunately, Rubinstein result holds only if we consider the restrictive case of linear orders. Indeed, Le Breton and Salles [6] have shown that the stability set can be empty if the individual preferences are complete preorders.

Theorem 3 (Le Breton and Salles [6]).

(i) For any integer $s$ with $k \leq 2 s-3$, the stability set is non-empty for any game for which $\nu(G)=s$ and any profile $(\succsim) \in \mathcal{R}^{n}$.

(ii) For any integer $s$ with $k \geq 2 s-1$, there exists a number of individuals $n$, a voting game $G=(N, W)$ for which $\nu(G)=s$ and a profile $(\succsim) \in \mathcal{R}^{n}$ such that the stability set of order one is empty.

(iii) Let $G(n, q)$ be a quota game for which $\nu(G)=\frac{k+2}{2}$. Then the stability set is non-empty for any profile $(\succsim) \in \mathcal{R}^{n}$.

Le Breton and Salles have shown in a counterexample that (iii) does not hold in general for an arbitrary voting game.

Actually, Le Breton and Salles propose an upper bound on the number of alternatives which guarantees the non emptiness of the

\footnotetext{
${ }^{1}$ We denote by $\lceil Y\rceil$ the smallest integer greater than or equal to $Y$.
} 
stability set. However, they do not state that there exists a profile such that the stability set is empty if the number of alternatives is greater than their bound. This result is partially resolved by Martin [8] for the particular case of quota games, who proposes a necessary and sufficient condition and a bound equal to $\left\lceil\frac{2 n}{n-q}\right\rceil$. Unfortunately, we are not able to present a similar necessary and sufficient condition for every proper voting game. We propose a sufficient condition and we show that the bound, $\theta$, is always greater than or equal to the one given by Le Breton and Salles. In contrast to Le Breton and Salles, in the construction of $\theta$, we do not use the Nakamura number.

\section{A new number for possibility theorems}

In this section, we introduce a new number, which characterizes the game, and leads to a sufficient condition for the non emptiness of the stability set. To compute it, we need a more precise definition of $\sigma$, a subset of $W$. First, we remove from $N$ all the dummy players, that is players $i$ such that:

$$
\forall S \ni i, S \in W \Rightarrow S \backslash\{i\} \in W
$$

This modifies the game $G(N, W)$ into a game $G\left(N^{\prime}, W^{\prime}\right)$, where each player is pivotal at least once.

In the computation of the Nakamura number, $\sigma$, a subset of coalitions, need not be ordered. We denote by $\gamma$ an ordered non empty subset of $W^{\prime} ; \gamma=\left(C_{\gamma(1)}, C_{\gamma(2)}, \ldots, C_{\gamma\left(t_{\gamma}\right)}\right), t_{\gamma}=|\gamma|$.

Definition 6. Let $\Gamma$ be the set of $\gamma$ 's that satisfy the following two conditions:

- a) For any player $i \in N$, there exist two successive coalitions, $C_{\gamma(\ell)}$ and $C_{\gamma(\ell+1)}$, in $\gamma$ such that $i \notin C_{\gamma(\ell)} \cup C_{\gamma(\ell+1)}{ }^{2}$

- b) If condition a) does not hold for some player $i$, this player belongs to exactly half of the coalitions in $\gamma$.

We define the new characteristic number for the game $G(N, W)$ by $\theta(G)$ :

$$
\theta(G)=\operatorname{Min}_{\gamma \in \Gamma}\{|\gamma|\}
$$

If $\Gamma$ is empty, by convention, we will assume that $\theta=\infty$.

${ }^{2}$ For $\ell=t_{\gamma}$, we will define $C_{\gamma(\ell+1)}$ by $C_{\gamma(1)}$. 
Thus, $\theta$, when it is finite, is the minimal number of coalitions we can order in such a way that each player either does not belong to two successive coalitions or belongs to one over two coalitions.

Example 3. The ordering of the coalitions in $\gamma$ is of crucial importance for the computation of $\theta$. Consider for example the following minimal winning coalitions $(N=5): C_{1}=\{1,2,3\}, C_{2}=\{2,3,4\}$, $C_{3}=\{1,4,5\}, C_{4}=\{3,4,5\}$, and $C_{5}=\{1,2,5\}$. Condition a) is not met for players 1 and 3 in the ordering $\gamma=\left(C_{1}, C_{2}, C_{3}, C_{4}, C_{5}\right)$. Nevertheless, we can prove that $\theta=5$ with the sequence $\gamma^{\prime}=$ $\left(C_{1}, C_{2}, C_{4}, C_{3}, C_{5}\right)$.

Example 4. Removing first dummy players is also important. Consider the weighted game $G(4,4,4,1 ; 7) \cdot \gamma=(\{1,2\},\{1,2,4\},\{1,3\}$ $,\{1,3,4\},\{2,3\},\{2,3,4\})$ could lead to $\theta=6$. This is misleading, as the preferences of player 4 never count in this weighted game. In fact, $\theta=\infty$.

Theorem 4. Let $G(N, W)$ be a voting game. If $k<\theta$, it is impossible to build a cycle with the domination of order one; Hence the stability set is non-empty for any preference profile in $\mathcal{R}^{n}$.

Proof. Suppose that $S(G,(\succsim))=\emptyset$ and $k<\theta$. Thus, there exists at least one cycle of length $k^{\star} \leq k$ for the profile $(\succsim)$ :

$$
x_{1} \succ \succ_{C_{1}} x_{2}, x_{2} \succ \succ_{C_{2}} x_{3}, \ldots, x_{k^{\star}-1} \succ \succ_{C_{k^{\star}-1}} x_{k^{\star}}, x_{k^{\star}} \succ \succ_{C_{k^{\star}}} x_{1} .
$$

Denote by $\gamma$ the sequence $\left(C_{1}, C_{2}, \ldots, C_{k^{\star}}\right)$. We first show that there exists an individual $i \in N$ such that:

- (A) $i \notin C_{\ell}$ implies $i \in C_{\ell-1} \cap C_{\ell+1}$.

- (B) There exist two successive coalitions in $\gamma$ such that $i \in C_{\ell} \cap$ $C_{\ell+1}$.

Assume the contrary: For all $i \in N$, either A) or B) is false, and at least one the two following statement is true:

- ᄀ(A): $i \notin C_{\ell} \cup C_{\ell+1}$ for some $\ell$.

- $\neg(B): i \in C_{\ell}$ implies $i \notin C_{\ell} \cap C_{\ell+1}$ for all $C_{\ell} \in \gamma$. 
If $\neg(A)$ is met for player $i$, condition $(a)$ for the definition of $\theta$ is satisfied. Similarly, if $\neg(B)$ and $A$ are both true for some $i$, this player belongs to exactly half of the coalitions in gamma and the condition (b) of the definition of $\theta$ is met. Thus, the sequence $\gamma$ defines an eligible $\theta^{\prime}<\theta$, a contradiction.

Thus, there exists $i \in N$ who belongs to two successive coalitions in $\gamma$, and belongs to $C_{\ell-1}$ and $C_{\ell+1}$ whenever he does not belongs to $C_{\ell}$. Assume, without loss of generality, that $i \in C_{k^{\star}-1} \cap C_{k^{\star}}$. Then,

$$
\left.\begin{array}{l}
x_{k^{\star}} \succ_{i} x_{1} \\
x_{k^{\star}-1} \succ_{i} x_{k^{\star}}
\end{array}\right\} \text { and, by transitivity, } x_{k^{\star}-1} \succ_{i} x_{1} .
$$

Then, two cases are possible: Either $i \in C_{k^{\star}-2}$ or $i \notin C_{k^{\star}-2}$. If $i \in$ $C_{k^{\star}-2}$, by transitivity, $x_{k^{\star}-2} \succ_{i} x_{1}$. If $i \notin C_{k^{\star}-2}$, as $i \in C_{k^{\star}-1}$, we get, by the definition of $\succ \succ$, that $x_{k^{\star}-2} \succeq x_{k^{\star}}$, and, by the transitivity of $\succ_{i}, x_{k^{\star}-2} \succ x_{1}$.

Consider now $C_{k^{\star}-3}$. If $i \in C_{k^{\star}-3}$, then $x_{k^{\star}-3} \succ_{i} x_{k^{\star}-2}$, and, by transitivity, $x_{k^{\star}-3} \succ_{i} x_{1}$. If $i \notin C_{k^{\star}-3}$, it cannot be that $i \notin C_{k^{\star}-2}$ by (A). Thus, as $x_{k^{\star}-2}$ dominates $x_{k^{\star}-1}$, we get $x_{k^{\star}-3} \succeq_{i} x_{k^{\star}-1}$, $x_{k^{\star}-1} \succ_{i} x_{1}$, and, at last, $x_{k^{\star}-3} \succ_{i} x_{1}$.

The fact that (A) holds for voter $i$ enables us to prove that any alternative $x_{2}, x_{3}, \ldots, x_{k^{\star}}$ dominates $x_{1}$, which is impossible as the preference of voter $i$ is supposed to be transitive. Thus, it is not possible to build that cycle, and the stability set is non empty.

Theorem 5. The greatest upper bound on the number of alternatives which guarantees the non-emptiness of the stability set proposed by Le Breton and Salles, $2 \nu-2$, is always less than or equal to $\theta$ for any voting game.

Proof. Assume on the contrary: There exists a game and an associated Nakamura number such that $2 \nu-2>\theta$. That is, $\nu>\frac{\theta}{2}+1$. Suppose now that $\theta$ is even. The minimal value of the Nakamura number is $\frac{\theta}{2}+2$. Consider now the collection of winning coalitions $\gamma=\left(C_{1}, C_{2}, \ldots, C_{\theta}\right)$ which implies the construction of $\theta$. If we select any $\nu-1$ winning coalitions in this collection, that is at least $\frac{\theta}{2}+1$, there exists an individual belonging to all these coalitions by the definition of the Nakamura number. Therefore, an individual $i$ belongs to the coalitions $C_{1}, C_{3}, C_{5}, \ldots, C_{\theta-1}, C_{\theta}$. In this case, there are no two 
successive winning coalitions $C_{\ell}, C_{\ell+1}$ such that $i \notin C_{\ell} \bigcup C_{\ell+1}$ and the condition $b$ ) is not met too, a contradiction of the definition of $\theta$.

If $\theta$ is odd, the minimal value for $\nu$ is $\frac{\theta+3}{2}$. Thus, $\nu-1=\frac{\theta+1}{2}>\frac{\theta}{2}$. There is always an individual belonging to any selection of $\nu-1$ coalitions in $\gamma$; Condition a) and b) cannot be met.

The following result seems to be important for the research of a necessary condition. It shows a correspondence between the bound for an arbitrary voting game and the one given by Martin [8] for the quota games. Therefore, we can think that $\theta$ is close to the highest bound implying the non-emptiness of the stability set.

Theorem 6. If a voting game with an associated $\theta$ is a quota game, then $\theta=\left\lceil\frac{2 n}{n-q}\right\rceil$.

Proof. First assume that $\theta<\frac{2 n}{n-q}$, that is, $(n-q) \theta<2 n$. Each voter does not belong to at least two coalitions in a sequence $\gamma$ that defines $\theta$, so $2 n$ is the minimal number for $\sum_{C_{j} \in \gamma}\left|N-C_{j}\right|$. On the other hand $\sum_{C_{j} \in \gamma}\left|N-C_{j}\right|$ must be lower to or equal than $(n-q) \theta$, a contradiction.

Secondly, let us construct a sequence of $\theta$ different coalitions such that conditions a) and b) are met.

- Case 1: $\theta=\frac{2 n}{n-q}$, with $n-q$ even. Thus, $(n-q) \theta=2 n$. The problem is equivalent to building $\theta$ coalitions $\bar{C}_{j}$ of size $n-q$ $\left(\bar{C}_{j}=N-C_{j}\right)$ such that, for all $i \in N$, there exists $\bar{C}_{\ell}$ for which $i \in \bar{C}_{\ell} \cup \bar{C}_{\ell+1}$. As $(n-q)$ is even, we can assign first $(n-q) / 2$ players to each coalitions such as:

$$
\begin{aligned}
& \left\{1, \ldots, \frac{n-q}{2}\right\} \in \bar{C}_{1} \\
& \left\{\frac{n-q}{2}+1, \ldots, 2 \frac{n-q}{2}\right\} \in \bar{C}_{2} \\
& \vdots \\
& \left\{(\theta-1) \frac{n-q}{2}+1, \ldots, \theta \frac{n-q}{2}\right\} \in \bar{C}_{\theta}
\end{aligned}
$$

To fill the remaining $(n-q) / 2$ position left in $\bar{C}_{j}$, add the players that belong to $\bar{C}_{j-1}$. Thus, all players appears twice in two successive coalitions. 
- Case 2: $\theta=\frac{2 n}{n-q}$, with $n-q$ odd. As $\theta(n-q)=2 n, \theta$ is even. First put player 1 in $\bar{C}_{1} \cup \bar{C}_{2}$, player 2 in $\bar{C}_{3} \cup \bar{C}_{4}, \ldots$ till player $\theta / 2$ in $\bar{C}_{\theta-1} \cup \bar{C}_{\theta}$. Thus, conditions a) is already fulfilled for players 1 to $\theta / 2 . n-\theta / 2$ players are left, and $(n-q-1)$ possibilities remain in the coalitions. As $(n-q-1)$ is even, we can use the same process as in Case 1. This is possible as $(n-q-1) \theta=(n-q) \theta-\theta=$ $2(n-\theta / 2)$.

- Case 3: $\theta=\left\lceil\frac{2 n}{n-q}\right\rceil>\frac{2 n}{n-q}$. If $(n-q)$ or $\theta$ is even, where are back to Cases 1 and 2. In fact, $\theta(n-q)=2 x>2 n$ and there are always enough rooms left. The case $(n-q)$ and $\theta$ odd remains ( e.g. $n-q=3, n=10$ and $\theta=7$ ). A solution is to first assign players 1 to $(\theta-1) / 2$ to coalitions 1 to $\theta-1$, as in the first step of Case 2. Next, $n-(\theta-1) / 2$ players must be assigned in two successive coalitions. There are $(n-q-1) \theta$ rooms left, but $(n-q-1) \theta=(n-q) \theta-\theta>2 n-\theta \geq 2 n-\theta-1=2(n-(\theta-1) / 2)$. We can use the same process as in Case 1 .

It remains to show that $\theta$ can be significatively different from $2 \nu-3$, the bound given by Le Breton and Salles.

Example 5. Consider the weighted game $G(11,3,3,3,3 ; 12) . \nu(G)=$ 3 , and $2 \nu-3=3$. As there is just one winning coalition without player $1, C=\{2,3,4,5\}, \theta=\infty$.

Example 6. Consider a game with 6 players and the following minimal winning coalitions: $C_{1}=\{2,3,6\}, C_{2}=\{1,3,4,6\}, C_{3}=$ $\{1,5,6\}, C_{4}=\{1,2,4,5\}, C_{5}=\{2,3,4,5\}, C_{6}=\{1,3,5\}$. One can check that $\nu(G)=3$ with $\sigma=\left\{C_{1}, C_{3}, C_{6}\right\}$, and $2 \nu-3=3$. One can also check that it is impossible to built a sequence $\gamma$ that satisfies condition a) and b) with five coalitions (hint: player 1 belongs to all the coalitions except $C_{1}$ and $C_{5}$, player 3 belongs to all the coalitions except $C_{3}$ and $C_{4}$, player 5 belongs to all the coalition except $C_{1}$ and $\left.C_{2}\right)$. Thus, $\theta=6$ with $\gamma=\left(C_{2}, C_{1}, C_{5}, C_{6}, C_{3}, C_{4}\right)$. Thus, we know that the stability set of this game will be non empty for $k=5$; This was impossible to guess with Le Breton and Salles' bound.

Example 7 proves that $k<\theta$ is not a necessary condition for the non-emptiness of the stability set. 
Example 7. Consider a game with 5 players and the following minimal winning coalitions: $C_{1}=\{1,2,3\}, C_{2}=\{2,3,4\}, C_{3}=\{3,4,5\}$, $C_{4}=\{1,4,5\}$. If we only consider these coalitions, we cannot built a sequence $\gamma$ which satisfies the conditions a) and b). Nevertheless, neither the definition we gave nor the proof of Theorem 1 involve the fact that we should only use minimal winning coalitions. Thus we can prove that $\theta=6$ with the sequence $\gamma=\left(C_{6}=\{1,2,3,5\}, C_{1}=\right.$ $\{1,2,3\}, C_{2}=\{2,3,4\}, C_{3}=\{3,4,5\}, C_{4}=\{1,4,5\}, C_{5}=\{1,2,4,5\}$. $4 \notin C_{6} \bigcap C_{1}, 5 \notin C_{1} \bigcap C_{2}, 1 \notin C_{2} \bigcap C_{3}, 2 \notin C_{3} \bigcap C_{4}$ and $3 \notin$ $C_{4} \cap C_{5}$. There is no other possible sequence to get $\theta=6$. Thus, for $k=6$, we may expect an empty stability set for some preference profiles. Assume that we have the following cycle:

$$
x_{1} \succ \succ_{C_{1}} x_{2} \succ \succ_{C_{2}} x_{3} \succ \succ_{C_{3}} x_{4} \succ \succ_{C_{4}} x_{5} \succ_{C_{5}} x_{6} \succ \succ_{C_{6}} x_{1}
$$

This cycle put some restrictions on the preferences of the players:

$$
\begin{aligned}
& x_{4} \succ_{1} x_{5} \succ_{1} x_{6} \succ_{1} x_{1} \succ_{1} x_{2} \\
& x_{5} \succ_{2} x_{6} \succ_{2} x_{1} \succ_{2} x_{2} \succ_{2} x_{3} \\
& x_{6} \succ_{3} x_{1} \succ_{3} x_{2} \succ_{3} x_{3} \succ_{3} x_{4} \\
& x_{2} \succ_{4} x_{3} \succ_{4} x_{4} \succ_{4} x_{5} \succ_{4} x_{6} \\
& x_{3} \succ_{5} x_{4} \succ_{5} x_{5} \succ_{5} x_{6} \succ_{5} x_{1}
\end{aligned}
$$

From this partial profile, we can observe that $x_{2} \succ_{C_{2}} x_{3}$ and $x_{6} \succ_{C_{1}}$ $x_{2}$. Since $x_{2} \succ \succ_{C_{2}} x_{3}, x_{6} \succsim x_{3} \forall i \in C_{2}$. This contradicts the fact that $x_{3} \succ_{4} x_{6}$. It is not possible to build a cycle for the dominance of order 1 if $k=6$.

Incidentaly, Example 7 raises the question of whether we could restrict ourselves to minimal winning coalitions in the computation of $\theta$. Unfortunately, contrary to the Nakamura number, Example 8 shows that we can't. The fact that $\theta=\infty$ when we consider minimal winning coalitions only does not guaranty the non emptiness of the stability set.

Example 8. Consider a 9-player game and the following minimal coalitions: $C_{1}=\{1,2,3,4,9\}, C_{2}=\{2,3,4,5,6\}, C_{4}=\{4,5,7,8,9\}$, $C_{5}=\{3,5,6,7,8\}, C_{6}=\{1,2,7\}$. If we only consider these coalitions, we cannot built a sequence $\gamma$ which satisfies the conditions 
a) and b). Nevertheless, we can prove that $\theta=6$ if we also consider the coalition $C_{3}=\{1,2,4,7\} \supset C_{6}$ since we can build a sequence $\gamma=\left(C_{1}, C_{2}, C_{3}, C_{4}, C_{5}, C_{6}\right)$ which mets conditions a) and b). $7,8 \notin C_{1} \bigcap C_{2}, 8,9 \notin C_{2} \bigcap C_{3}, 3,6 \notin C_{3} \bigcap C_{4}, 1,2 \notin C_{4} \bigcap C_{5}$, $4 \notin C_{5} \bigcap C_{6}, 5 \notin C_{1} \bigcap C_{6}$. Consider the following preferences over 6 alternatives:

$$
\begin{aligned}
& x_{6} \succ_{1} x_{1} \sim_{1} x_{3} \sim_{1} x_{5} \succ_{1} x_{2} \succ_{1} x_{4} \\
& x_{6} \succ_{2} x_{1} \sim_{2} x_{5} \succ_{2} x_{2} \succ_{2} x_{3} \succ_{2} x_{4} \\
& x_{1} \sim_{3} x_{5} \succ_{3} x_{2} \sim_{3} x_{4} \sim_{3} x_{6} \succ_{3} x_{3} \\
& x_{1} \succ_{4} x_{2} \sim_{4} x_{6} \succ_{4} x_{3} \succ_{4} x_{4} \succ_{4} x_{5} \\
& x_{2} \sim_{5} x_{4} \succ_{5} x_{1} \sim_{5} x_{3} \sim_{5} x_{5} \succ_{5} x_{6} \\
& x_{2} \sim_{6} x_{5} \succ_{6} x_{1} \sim_{6} x_{3} \sim_{6} x_{4} \sim_{6} x_{6} \\
& x_{3} \succ_{7} x_{2} \sim_{7} x_{4} \succ_{7} x_{5} \succ_{7} x_{6} \succ_{7} x_{1} \\
& x_{4} \succ_{8} x_{1} \sim_{8} x_{2} \sim_{8} x_{3} \sim_{8} x_{5} \succ_{8} x_{6} \\
& x_{1} \sim_{9} x_{4} \succ_{9} x_{2} \sim_{9} x_{3} \sim_{9} x_{5} \sim_{9} x_{6}
\end{aligned}
$$

There are only 6 dominance relations for this profile (see Table 1 in the appendix for all the details):

$$
x_{1} \succ_{C_{1}} x_{2}, x_{2} \succ_{C_{2}} x_{3}, x_{3} \succ_{C_{3}} x_{4}, x_{4} \succ_{C_{4}} x_{5}, x_{5} \succ_{C_{5}} x_{6}, x_{6} \succ_{C_{6}} x_{1}
$$

For all $\ell=1, \ldots, 6$, one can check that for all $i \in C_{\ell}, x_{\ell-1} \succsim_{i} x_{\ell+1}$; in turns, $x_{\ell} \succ \succ_{C_{\ell}} x_{\ell+1}{ }^{3}$. Thus, the stability set is empty.

\section{Conclusion}

The main contribution of this paper is to show that the non emptiness of the stability set depends upon a new number, $\theta$, that is not related to the Nakamura number. Example 5 and 6 have shown that the two numbers, $\theta$ and $\nu$, can be different, but we don't know yet whether they can be arbitrarily different. Another open issue is to find an algorithm for the computation of $\theta$; Some of the examples we provide here prove to be tricky. At least, the main unsolved problem is the prove whether $\theta$ is really the right bound, that is, to build for any game an empty stability set if $k=\theta$. The fact that $\theta$ gives back the right conditions for quota games is encouraging, but Example 7 shows that we may have to compute $\theta$ in a different way to get a necessary and sufficient condition.

\footnotetext{
${ }^{3}$ Clearly, when $\ell=6, \ell+1=1$
} 


\section{References}

1. M.J.A. Condorcet, (Marquis de). Essai sur l'application de l'analyse à la probabilité des décisions rendues à la pluralité des voix. Imprimerie Royale, Paris, 1785.

2. J. Ferejohn and D. Grether. On a class of rational social decision procedures. Journal of Economic Theory, 8:471-482, 1974.

3. D.B. Gillies. Solutions to general non-zero-sum games. In A.W. Tucker and R.D. Luce, editors, Contributions to the theory of games, volume 4. Princeton University Press, Princeton, 1959.

4. J. Greenberg. Consistent majority rules over compact sets of alternatives. Econometrica, 47:627-636, 1979.

5. M. Le Breton. On some combinatorial problems arising in the theory of voting games. Mathematical Social Sciences, 19:179-193, 1990.

6. M. Le Breton and M. Salles. The stability set of voting games: classification and genericity results. International Journal of Game Theory, 19:111-127, 1990.

7. S. Li. Stability of voting games. Social Choice and Welfare, 10:51-56, 1993.

8. M. Martin. A note on the non-emptiness of the stability set. Social Choice and Welfare, 17:559-566, 2000.

9. N. Miller. A new solution set for tournaments and majority voting: further graph theoretical approaches to the theory of voting. American Journal of Political Sciences, 24:68-96, 1980.

10. H. Moulin. Choosing from a tournament. Social Choice and Welfare, 3:271-292, 1986.

11. K. Nakamura. The vetoers in a simple game with ordinal preferences. International Journal of Game Theory, 8:55-61, 1979.

12. B. Peleg. Consistent voting systems. Econometrica, 46:153-161, 1978.

13. A. Rubinstein. Stability of decision systems under majority rule. Journal of Economic Theory, 23:150-159, 1980. 


\section{Appendix}

Table 1. The winning coalitions for Example 8

\begin{tabular}{|c|c|c|c|c|}
\hline Option & $\succ$ & $\sim$ & $\prec$ & Option \\
\hline$x_{1}$ & $\mathbf{1 , 2 , 3 , 4 , 9}$ & 8 & $5,6,7$ & $x_{2}$ \\
$x_{1}$ & $2,3,4,9$ & $1,5,6,8$ & 7 & $x_{3}$ \\
$x_{1}$ & $1,2,3,4$ & 6,9 & $5,7,8$ & $x_{4}$ \\
$x_{1}$ & 4,9 & $1,2,3,5,8$ & 6,7 & $x_{5}$ \\
$x_{1}$ & $4,5,8,9$ & 6 & $\mathbf{1 , 2 , 3 , 7}$ & $x_{6}$ \\
$x_{2}$ & $\mathbf{2 , 3 , 4 , 5 , 6}$ & 8,9 & 1,7 & $x_{3}$ \\
$x_{2}$ & $1,2,4,6$ & $3,5,7$ & 8,9 & $x_{4}$ \\
$x_{2}$ & $4,5,7$ & $6,8,9$ & $1,2,3$, & $x_{5}$ \\
$x_{2}$ & $5,6,7,8$ & $3,4,9$ & 1,2, & $x_{6}$ \\
$x_{3}$ & $\mathbf{1 , 2 , 4 , 7}$ & 6 & $3,5,8,9$ & $x_{4}$ \\
$x_{3}$ & 4,7 & $1,5,8,9$ & $2,3,6$ & $x_{5}$ \\
$x_{3}$ & $5,7,8$ & 6,9 & $1,2,3,4$ & $x_{6}$ \\
$x_{4}$ & $\mathbf{4 , 5 , 7 , 8 , 9}$ & & $1,2,3,6$ & $x_{5}$ \\
$x_{4}$ & $5,7,8,9$ & 3,6 & $1,2,4$ & $x_{6}$ \\
$x_{5}$ & $\mathbf{3 , 5 , 6 , 7 , 8}$ & 9 & $1,2,4$, & $x_{6}$ \\
\hline
\end{tabular}

For each pair of alternatives $\left(x_{i}, x_{j}\right)$, we indicate the voters who prefer $x_{i}$ to $x_{j}$ in the first column, the voters who are indifferent in the second column, and the voters who prefer $x_{j}$ to $x_{i}$ in the third column. The winning coalitions are outlined in bold. 